\title{
The Generalized Extreme Value Model for Seismic Hazard Estimation of Potential Source Area based on Maximum Information Entropy Principle
}

Luchuan Ren, Zhe Liu, Jianwei Tian

Institute of Disaster Prevention, China Earthquake Administration, Beijing East Yanjiao 101601, China

\section{基于最大信息摘原理构建潜源区强震危险性估 计广义极值模型}

任鲁川, 刘哲, 田建伟

中国地震局防灾科技学院，北京东燕郊 101601

\begin{abstract}
The author proposes a method to establish the generalized extreme value model for strong earthquake hazard estimation of a potential source area, based on the maximum information entropy principle and corresponding constraint conditions, and then selects the Ryukyu trench subduction zone as a potential seismic source area for case study. The results show: Considering that the Weibull distribution, among the three kinds of generalized extreme value distribution, has limited upper extreme point, and the upper bound magnitude of a potential seismic source area should be limited, therefore the Weibull distribution model should be selected to establish the generalized extreme value model for strong earthquake hazard estimation; Under the condition that the upper bound magnitude of a potential seismic source area be limited, and in according with the maximum information entropy principle, the probability density function of the maximum magnitude of a potential seismic source area, in
\end{abstract}

each time interval, can be expressed as a general formula with three parameters, and these parameters can be calculated from the estimated values of shape parameter, location parameter and scale parameter of the corresponding Weibull distribution.

Keywords: maximum information entropy principle; generalized extreme value model; potential seismic source area; seismic hazard estimation

摘要

提出在符合最大信息熵原理并满足相应约束条件 的情况下, 构建潜源区强震危险性估计广义极值模 型的方法, 并选琉球海沟俯冲带潜源区进行案例研 究。结论如下: 因为潜源区震级上限为有限值, 所 以构建潜源区强震危险性估计广义极值模型应取 Weibull 分布; 在满足潜源区震级上限为有限值的 约束条件下, 可用符合最大信息熵原理的概率分布 通式表示潜源区各个等长时段最大震级的概率分 布函数; 该通式含三个参数, 其值可由上述 Weibull 分布的形状参数、位置参数和尺度参数估计值求 
Risk Analvsis and Crisis Response in Biq Data Era (RAC-16)

得。

关键词：最大信息熵原理；广义极值模型；潜 源区; 强震危险性估计

\section{1. 引言}

目前有两类潜源区强震危险性估计方法, 一是地震统计学方法, 一是特征地震方法[1]。 前者基于震级-频度关系, 通过统计分析历史 史料记载和地震仪记录的潜源区地震数据, 估 计强震危险性[2][3]; 依据最大信息熵原理可 以解释震级-频度关系的物理意义[4-10]。后者 基于古地震或历史地震遗迹考察, 依据推断的 断层滑动速率，估计该断层特征地震的重现 时间; 通过描述断层行为的简单力学模式可以 定性解释强震危险性估计结果 [10]。两类方法 都有局限。地震统计学方法在大震级区段往往 出现所谓 “掉头” 现象 $[11,12]$ 。由于野外环境 和考察条件的限制, 许多潜源区难以采用特征 地震方法。近年，广义极值模型逐渐被应用到 潜源区强震危险性估计, 这类模型不仅无需先 验地选定震级上限, 可以更充分地利用大震级 区段的信息[3,13-18], 但其所蕴含的物理意义 未见深入分析和探讨。

本文首先介绍熵的有关概念和最大信息 熵原理, 然后讨论在有关地震学原理和最大信 息熵原理的约束下, 构建潜源区强震危险性估 计广义极值模型的方法, 最后选琉球海沟俯冲 带潜源区进行案例研究。

\section{2. 熵的概念和最大信息熵原理}

\section{1. 熵的有关概念}

1854年, 克劳修斯(Clausius)在热力学研究 中引入了一个新的态函数一熵(entropy), 被称 为克劳修斯熵或热力学熵。对于无限小过程, 热力学第二定律可以表示成商的微分形式

$$
d S \geq \frac{d Q}{T}
$$

式中, 符号 $\mathrm{S}$ 表示熵, $\mathrm{Q}$ 表示热量, $\mathrm{T}$ 表示 温度, 等号对应于可逆过程, 不等号对应于不 可逆过程[19]。1896 年玻尔兹曼(Boltzmann )
建立了熵与系统宏观态所对应的可能的微观 态数目的联系: $S \propto \ln W$, 式中 $\mathrm{S}$ 表示熵, $\mathrm{W}$ 表示微观态数目。1900 年, 普朗克 (Planck) 引进了玻尔兹曼常量 $\mathrm{K}$, 给出玻尔兹曼-普朗 克公式

$$
S=K \ln W
$$

此式所定义的熵被称为统计熵。统计熵揭示了 热力学第二定律的统计本质: 熵增加原理表示 孤立系统中热力学过程的方向性, 相应于系统 从热力学概率小的状态向热力学概率大的状 态过渡, 平衡态的热力学概率最大, 对应于统 计熵为极大值的状态, 熵自发减小的过程不是 绝对不可能, 只不过概率非常小而已。1948 年仙农 (Shannon) 发表了《通信的数学理论》, 使用概率方法, 奠定了现代信息论的基础。他 提出了被称为仙农熵的信源信息熵概念, 用

$$
H(X)=-\sum_{i} P\left(a_{i}\right) \log _{2} P\left(a_{i}\right)
$$

表示信源输出每个消息所提供的平均信息量, 其中 ai 为信源可能取的消息, $P$ (ai) 为选择 信源符号 ai 作为消息的先验概率。1957 年詹 尼斯 (Jaynes ) 将信息熵引入统计力学, 给出 的信息熵定义:

$$
S=-k \sum_{i} P_{i} \ln P_{i}
$$

此式比仙农商表达式多一比例系数[20-22]。

\section{2. 最大信息熵原理}

1957 年杰尼斯 (E.T.Jaynes) 提出最大信 息熵原理: 在只掌握部分信息的情况下, 对未 知的分布形态做推断, 应该选择符合约束条件 同时信息熵最大的概率分布, 任何其他选择都 意味着添加额外的约束, 而且根据所掌握的 信息这些额外的约束是无法做出的。最大信 息熵原理是一种选择随机变量统计特性最 符合客观情况的一个准则。一般而言, 随机 量的概率分布是很难测定的, 通常只能测得 其各种均值 (如数学期望、方差等) 或已知 某些限定条件下的值 (如峰值、取值个数 等）, 与这些测值相符合的分布可有多种以 至无穷多种, 但其中仅有一种分布的信息熵 
最大。选用这种具有最大信息摘的分布作为随 机变量的分布, 是一种有效的处理方法和准则 [22]。

\section{3. 基于最大信息摘原理的连续型随机变量 分布}

给定约束条件, 由最大信息熵原理求 “最 佳” 概率分布是一求解条件极值问题。设 $\mathrm{X}$ 为连续随机变量, $p(x)$ 为概率密度函数, 若 $\mathrm{X}$ 的若干函数 $\mathrm{fm}(\mathrm{x})$ 的数学期望满足约束条件

$$
\begin{gathered}
\int p(x) d x=1 \\
\int p(x) f_{m}(x) d x=C_{m}
\end{gathered}
$$

$\mathrm{m}=1,2, \ldots, \mathrm{M}, \mathrm{Cm}$ 为常数。信息熵表达式 为:

$$
H(x)=-\int p(x) \log p(x) d x
$$

$$
\text { 令 }
$$

$$
\begin{aligned}
G(x)= & H(x)+\left(\lambda_{0}+1\right)\left[\int p(x) d x-1\right] \\
& +\sum_{m=1}^{M} \lambda_{m}\left[\int p(x) f_{m}(x) d x-C_{m}\right]
\end{aligned}
$$

利用拉格朗日乘子法可导出满足随机变量 X 的信息熵 $\mathrm{H}$ 最大的概率分布：

$$
p(x)=\exp \left[\lambda_{0}+\sum_{m=1}^{M} \lambda_{m} f_{m}(x)\right]
$$

可见，基于最大信息熵原理，依据上述约束条 件, 连续型随机变量 $\mathrm{X}$ 的概率密度分布函数的 通式可以表示为 $e$ 的负指数次幂的形式 [23,24]。

\section{3. 潜源区强震危险性估计广义极值模型构建}

本节论述将广义极值建模原理与最大信 息熵原理 $[22,25]$ 相结合构建潜源区强震危险 性估计模型的方法。广义极值分布（the generalized extreme value distributions) 为:

$$
F(x)=\exp \left[-\left(1+\xi \frac{x-\mu}{\sigma}\right)^{-1 / \xi}\right]
$$$$
1+\xi(x-\mu) / \sigma>0
$$

式中 $\xi$ 为形状参数 (shape parameter) $\mu$ 为 位置参数 (location parameter), $\sigma$ 为尺度参数 (scale parameter), $\mu, \xi$ 定义域为实数域 $\mathrm{R}$, $\sigma>0 。 \xi=0$ 时为 Gumbel 分布, $\xi>0$ 时为 Frechet 分布, $\xi<0$ 时为 Weibull 分布。相应 的概率密度函数为

$$
p(x)=\frac{1}{\sigma}\left(1+\xi \frac{x-\mu}{\sigma}\right)^{-(1+1 / \xi)} \exp \left[-\left(1+\xi \frac{x-\mu}{\sigma}\right)^{-1 / \xi}\right]
$$

迄今地震仪器监测记录到的震级最大地 震是矩震级 9.5 级的 1960 年 5 月 22 日智利大 地震。特定潜在震源区所发生地震的震级上限 必定为有限值, 一般认为不会超过矩震级 10 级。考虑到三类广义极值分布中仅 Weibull 分 布具有有限上端点, 所以我们构建强震危险 性估计的广义极值模型应取 Weibull 分布。 为下文处理的方便, 令 $\alpha=1 / \xi, \mu-\alpha \sigma=\delta, \alpha \sigma=\beta$, (2-1) 和 (2-2) 变为

$$
\begin{aligned}
& \mathrm{F}(\mathrm{x})=\exp \left[-\left(\frac{\mathrm{x}-\delta}{\beta}\right)^{-\alpha}\right], \alpha<0 \text { 和 } \beta<0 \quad \text { (3-3) } \\
& \mathrm{p}(\mathrm{x})=\frac{\alpha}{\beta}\left(\frac{\mathrm{x}-\delta}{\beta}\right)^{-\alpha-1} \exp \left[-\left(\frac{\mathrm{x}-\delta}{\beta}\right)^{-\alpha}\right], \quad \mathrm{x}<\delta
\end{aligned}
$$

选定某一潜源区, 再选定一定时限并将 之划分为一系列相等的时段, 提取各个时段 的最大震级样本, 由这些样本拟合求得最大 震级的概率密度分布, 它应能表示为式 (2-4) 的 Weibull 分布的形式, 否则就说明 潜源区界定或获取最大震级样本的方法不 合理。另一方面, 可以认为上述最大震级的 概率密度分布应满足最大信息熵原理及上 述约束条件, 这意味着它也可表示为式 (2-8) 的形式。取式 (2-8) 前三项, 则

$p(x)=\exp \left(\lambda_{0}+\lambda_{1} f_{1}(x)+\lambda_{2} f_{2}(x)\right)$

取式（3-4）和（3-5）的自然对数, 得到 $\ln p(x)=\ln \left(\frac{\alpha}{\beta}\right)-(\alpha+1) \ln \left(\frac{x-\delta}{\beta}\right)-\left(\frac{x-\delta}{\beta}\right)^{-\alpha}$ 
Risk Analvsis and Crisis Response in Biq Data Era (RAC-16)

$\ln p(x)=\lambda_{0}+\lambda_{1} f_{1}(x)+\lambda_{2} f_{2}(x)$

令上两式相等, 则

$$
\begin{aligned}
\lambda_{0} & =\ln \left(\frac{\alpha}{\beta}\right) \\
\lambda_{1} & =-(\alpha+1) \\
\lambda_{2} & =-1 \\
f_{1}(x) & =\ln \left(\frac{x-\delta}{\beta}\right) \\
f_{2}(x) & =\left(\frac{x-\delta}{\beta}\right)^{-\alpha}
\end{aligned}
$$

\section{4. 应用案例}

以琉球海沟俯冲带为潜源区 $\left(22^{\circ}\right.$ $\left.\mathrm{N}-32.5^{\circ} \mathrm{N}, 120.5^{\circ} \mathrm{E}-133^{\circ} \mathrm{E}\right)$ 进行案例研究 （图 1)。琉球海沟俯冲带属于沟-弧-盆体系, 其中的 “沟” 指的是琉球海沟, 它是沟-弧-盆 体系与大洋盆地的天然分界线, 在地貌上表现 为岛坡坡麓的深沟, 它是一条向东南凸出, 向 西北倾没的弧形海沟, 呈环带状环绕琉球岛弧 延伸, 北端以九州-帛琉海岭为界, 南端位于 台湾岛中部外海, 总长约 1350 米, 平均宽度 $60 \mathrm{~km}$, 平均水深在 6000 米以上, 最大水深 $7781 \mathrm{~m}$ 。琉球海沟的西坡是具有大陆性质琉球 岛弧, 是由琉球诸岛形成的岛链, 称之为琉球 岛弧, 北起九州岛的南部, 南到台湾岛东部,

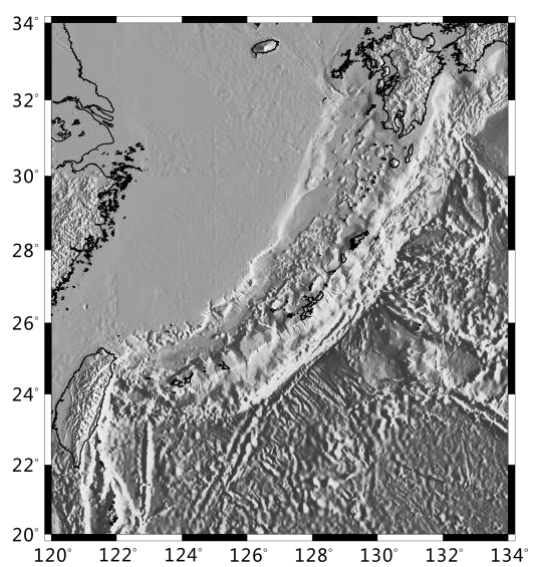

图 1. 琉球海沟俯冲带地理位置

全长 1200 公里, 属于双列岛弧, 内弧主要是
古琉球火山带形成的一条水下火山脊, 外弧是 琉球群岛的主体, 地貌分布格局主要由三个构 造--琉球群岛隆褶带, 弧前盆地和八重山海脊 带控制。琉球岛弧的西侧是冲绳海槽, 为一正 在发育的弧后裂谷盆地, 目前仍是大陆地壳。

历史地震记录取自美国地质调查局网站 (www.usgs.gov), 时限 1900-2010 年。以 10 年 为一时间时段, 得到 11 个时间时段的最大震 级数据序列（表 1)。对 11 个震级数据进行广 义极值拟合, 得到广义极值分布三个参数的极 大似然估计值及其标准差 (表 2)。形状参数 $\xi$ 的估计值是负值, 说明估计得到的分布形式 为 Weibull 分布。诊断 (图 2) 结果显示: 概 率和分位数的诊断图中 (图 $2 \mathrm{a}$, 图 2b) 的数据 点近似与直线拟合, 说明模型估计值与经验分 析值趋于一致; 重现水平曲线 (图 $2 \mathrm{c}$ ) 上端 逐渐趋向一有限值, 说明估计所得的模型属于 广义极值三种分布中的 Weibull 分布; 概率密 度曲线的估计与直方图 (图 2d) 具有一定程 度的拟合度。总之, 诊断结果不能否定广义极 值模型拟合的适用性。

表 1. 最大震级数据（时限 1900-2010, 时段 10 年)

\begin{tabular}{cc}
\hline 时段 & 最大震级(Mw) \\
\hline $1900-1910$ & 8.1 \\
$1910-1920$ & 8.2 \\
$1920-1930$ & 7.7 \\
$1930-1940$ & 7.9 \\
$1940-1950$ & 7.5 \\
$1950-1960$ & 7.8 \\
$1960-1970$ & 7.5 \\
$1970-1980$ & 7.4 \\
$1980-1990$ & 7.4 \\
$1990-2000$ & 7.7 \\
$2000-2010$ & 7.1 \\
\hline
\end{tabular}

表 2. 广义极值分布三个参数的极大似然对数估计 值及其标准差

\begin{tabular}{cccc}
\hline & 位置参数 & 形状参数 & 尺度参数 \\
\hline 估计值 & 7.56 & -0.32 & 0.31 \\
标准差 & 0.11 & 0.29 & 0.08 \\
\hline
\end{tabular}


将 Weibull 分布的三个参数的估计值代入 上限震级计算公式 $z_{0}=\mu-\sigma / \xi$, 可得到琉 球海沟俯冲带上限震级估计值为 8.53 级。将 Weibull 分布的三个参数估计值代入 $\alpha=1 / \xi$, $\mu-\alpha \sigma=\delta, \alpha \sigma=\beta$, 再将计算所得值代入式 (3-8) - (3-12), 由公式 (3-5), 得到琉球海沟俯 冲带满足最大熵原理的最大震级分布的概率 密度函数为:

$$
p(x)=\exp \left[1.17+2.13 \ln \left(\frac{x-8.53}{-0.97}\right)-\left(\frac{x-8.53}{-0.97}\right)^{3.13}\right]
$$
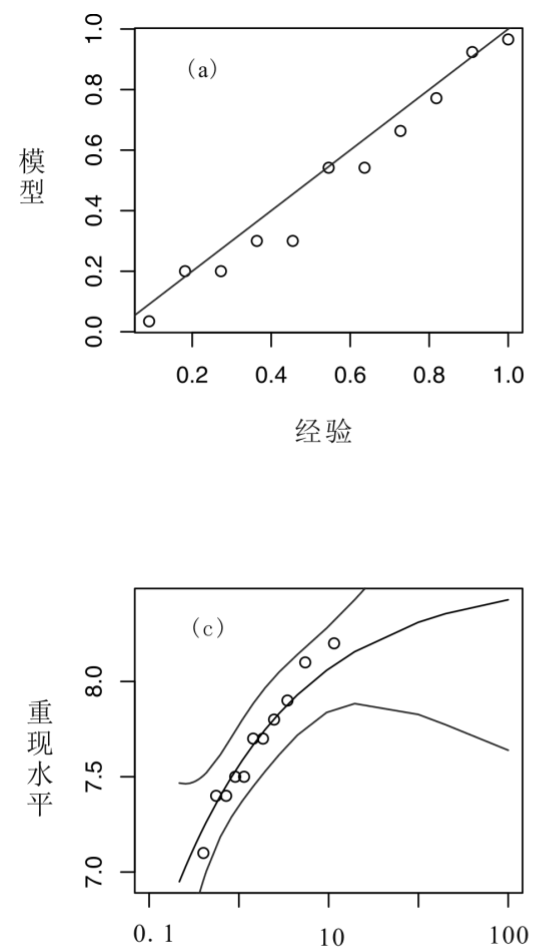

重现期
(2)潜源区各个时段最大震级的概率分布 密度函数可用符合最大信息熵原理的包含三 个参数的概率分布通式表示, 且这三个参数可 用估计得到的 Weibull 分布中的形状参数、位 置参数和尺度参数值求得。
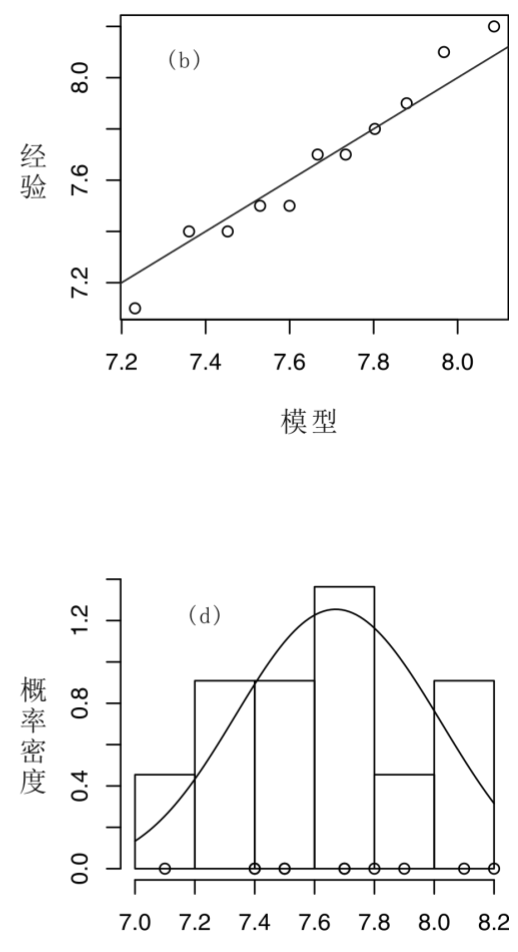

震级

图 2. 广义极值模型拟合最大震级数据的诊断图。(a)概率图, (b)分位数图, (c)重现水平图 (d) 概率密度图

\section{5. 结论}

(1) 鉴于所有三类广义极值分布中仅只 Weibull 分布具有有限的上端点, 再考虑到潜 源区震级上限必定为有限值, 构建强震危险性 估计的广义极值模型应选取 Weibull 分布模 型。

\section{致谢}

本研究得到了国家自然科学基金项目 (No. 41276020) 和中央高校基本科研业务费 （ZY20160312）、中央高校基本科研业务费 (ZY20160311) 的资助。 


\section{参考文献}

[1] 任鲁川, 霍振香, 洪明理.耦合潜源参数 不确定性效应的地震海啸危险性分析一 原理与方法.海洋预报,12(6): 7-13,2014.

[2] Cosentino P, Ficarra V, Luzio D. Truncated exponential frequency-magnitude relationship in earthquake statistics. Bulletin of the Seismological Society of America, 67(6):1615-1623,1977.

[3] Pisarenko V F, Sornette A, Sornette D, et al.Characterization of the Tail of the Distribution of Earthquake Magnitudes by Combining the GEV and GPD Descriptions of Extreme Value Theory. Pure \& Applied Geophysics, 171(8):1599-1624,2014.

[4] J. B. Berrill, R. O. Davis. Maximum entropy and the magnitude distribution. Bulletin of the Seismological Society of America, (5):1823-1831,1980.

[5] Shen P Y, Mansinha L. On the principle of maximum entropy and the earthquake frequency-magnitude relation. Geophysical Journal of the Royal Astronomical Society, 74(3):777-785,1983.

[6] 成尔林,张美芳.最大熵原理在地震重复关 系中的应用。地震研究, 10(2) : 139-144,1987.

[7] 彭美煊. 最大熵原理和综合概率法在计 算地震年发生率和平均重现期中的应用. 地震, (3):38-43,1993.

[8] 冯利华. 最大熵原理与地震频度一震级 关系. 地震地质, 25(2):260-265,2003.

[9] Angelo De Santis, Gianfranco Cianchini, Paolo Favali, Laura Beranzoli and Enzo Boschi.The Gutenberg-Richter Law and Entropy of Earthquakes: Two Case Studies in Central Italy, Bulletin of the Seismological Society of America, 101:1386-1395, 2011.

[10] S.G.Wesnousky,刘新美. 古登堡-里克特分 布还是特征地震分布. 世界地震译丛(5): 43-62,1995.

[11] 胡聿贤. 地震安全性评价技术教程[M] 北京：地震出版社.1999.

[12] Raschke M. Modeling of magnitude distributions by the generalized truncated exponential distribution. Journal of Seismology, 19(1):1-7,2014.

[13] Pisarenko V F, Sornette A, Sornette D, et al. New Approach to the Characterization of $M$ max and of the Tail of the Distribution of Earthquake Magnitudes. Pure \& Applied Geophysics, 165(5):847-888,2008.

[14] Raschke M. Comment on Pisarenko et al. "Characterization of the Tail of the
Distribution of Earthquake Magnitudes by Combining the GEV and GPD Descriptions of Extreme Value Theory". Pure \& Applied Geophysics. 112(1):1-7,2015.

[15] 钱小仕, 王福昌, 曹桂荣, 任晴晴. 广义 极值分布在地震危险性分析中的应用. 地震研究, 35(1): 73-78,2012.

[16] 钱小仕, 王福昌, 盛书中. 基于广义帕累 托分布的地震震级分布尾部特征分. 地 震学报, 35(3):341-350,2013a.

[17] 钱小仕, 蔡晓光, 任晴晴.中国大陆活动 地块边界带强震震级分布特征研究. 地震 工程与工程动,33(1):212-220,2013b.

[18] 任晴晴, 钱小仕, 赵玲玲, 霍振香. 中国 大陆活动地块边界带最大震级分布特征 研究.地震, 33(3), 67-76,2013.

[19] 王竹溪. 《热力学简程》, 北京: 人民教 育出版社, 1964.

[20] 任鲁川. 灾害熵: 概念引入及应用案例. 自然灾害学报, 02(2):26-31,2000.

[21] 李鹤龄. 信息熵、玻尔兹曼摘以及克劳修 斯熵之间的关系——兼论玻尔兹曼熵和 克劳修斯熵是否等价。大学物理, 23(12):37-40,2004.

[22] 石峰, 莫忠息. 《信息论基础》, 武昌: 武 汉大学出版社, 2002 .

[23] 李宪东. 基于最大熵原理的确定概率分 布的方法研究. 华北电力大学 (北 京),2008. .

[24] 余海, 陈勇, 周钦. 基于最大熵原理的风 险分析先验概率分布确定方法. 中国科 技信息, (4):276-277,2008.

[25] Stuart Coles. An Introduction to Statistical Modeling of Extreme Values. Springer-Verlag London limited. Printed in Great Britain,200. 